\title{
Characterizations of Besov-Type and Triebel-Lizorkin-Type Spaces by Differences
}

\author{
Douadi Drihem \\ Department of Mathematics, Laboratory of Pure and Applied Mathematics, M'Sila University, \\ P.O. Box 166, M'Sila 28000, Algeria
}

Correspondence should be addressed to Douadi Drihem, douadidr@yahoo.fr

Received 10 December 2010; Accepted 10 February 2011

Academic Editor: Hans Triebel

Copyright (C) 2012 Douadi Drihem. This is an open access article distributed under the Creative Commons Attribution License, which permits unrestricted use, distribution, and reproduction in any medium, provided the original work is properly cited.

We present characterizations of the Besov-type spaces $B_{p, q}^{s, \tau}$ and the Triebel-Lizorkin-type spaces $F_{p, q}^{s, \tau}$ by differences. All these results generalize the existing classical results on Besov and TriebelLizorkin spaces by taking $\tau=0$.

\section{Introduction}

The $B_{p, q}^{s, \tau}$ spaces and $F_{p, q}^{s, \tau}$ spaces have been studied extensively in recent years. When $\tau=0$ they coincide with the usual function spaces $B_{p, q}^{s}$ and $F_{p, q}^{s}$, respectively, studied in detail by Triebel in [1-3]. When $s \in \mathbb{R}, \tau \in[0, \infty)$ and $1 \leq p, q<\infty$ the $B_{p, q}^{s, \tau}$ spaces were first introduced by El Baraka in $[4,5]$. In these papers, El Baraka investigated embeddings as well as LittlewoodPaley characterizations of Campanato spaces. El Baraka showed that the spaces $B_{p, q}^{s, \tau}$ cover certain Campanato spaces, studied in [6,7]. Later on, Drihem gave in [8] a characterization for $B_{p, q}^{s, \tau}$ spaces by local means and maximal functions. For a complete treatment of $B_{p, q}^{s, \tau}$ spaces and $F_{p, q}^{s, \tau}$ spaces we refer the reader the work of Yuan et al. [9]. Yang and Yuan, in [1012], have introduced the scales of homogeneous Besov-Triebel-Lizorkin-type spaces $\dot{B}_{p, q}^{s, \tau}$ and $\dot{F}_{p, q}^{s, \tau}(p \neq \infty)$, which generalize the homogeneous Besov-Triebel-Lizorkin spaces $\dot{B}_{p, q}^{s, \tau}, \dot{F}_{p, q}^{s, \tau}$ and established the relation between $\dot{F}_{p, q}^{s, \tau}$ and $Q_{\alpha}$ spaces. See also [13] for further results.

Our main purpose in this paper is to characterize these function spaces by differences. These results are a generalization of some results given in [17], and [9, Chapter 4, Section 4.3]. All these results generalize the existing classical results on Besov spaces and Triebel-Lizorkin spaces by taking $\tau=0$.

The paper is organized as follows. Section 2.1 collects fundamental notation and concepts and Section 2.2 covers results from the theory of these function spaces. Some 
necessary tools are given in Section 3. These results are used in Section 4 to obtain the characterization of $B_{p, q}^{s, \tau}$ spaces and $F_{p, q}^{s, \tau}$ spaces by differences.

\section{Preliminaries}

\subsection{Notation and Conventions}

As usual, $\mathbb{R}^{n}$ the $n$-dimensional real Euclidean space, $\mathbb{N}$ the collection of all natural numbers, and $\mathbb{N}_{0}=\mathbb{N} \cup\{0\}$. The letter $\mathbb{Z}$ stands for the set of all integer numbers. For a multi-index $\alpha=\left(\alpha_{1}, \ldots, \alpha_{n}\right) \in \mathbb{N}_{0}^{n}$, we write $|\alpha|=\alpha_{1}+\cdots+\alpha_{n}$ and $D^{\alpha}=\partial^{|\alpha|} / \partial x_{1}^{\alpha_{1}} \cdots \partial x_{n}^{\alpha_{n}}$. For $v \in \mathbb{Z}$, let $B_{v}$ be the ball of $\mathbb{R}^{n}$ with radius $2^{-v}$ and $v^{+}=\max \{v, 0\}$. The Euclidean scalar product of $x=\left(x_{1}, \ldots, x_{n}\right)$ and $y=\left(y_{1}, \ldots, y_{n}\right)$ is given by $x \cdot y=x_{1} y_{1}+\cdots+x_{n} y_{n}$. We denote by $|\Omega|$ the $n$-dimensional Lebesgue measure of $\Omega \subseteq \mathbb{R}^{n}$. For any measurable subset $\Omega \subseteq \mathbb{R}^{n}$ the Lebesgue space $L^{p}(\Omega), 0<p \leq \infty$ consists of all measurable functions for which

$$
\begin{gathered}
\left\|f \mid L^{p}(\Omega)\right\|=\left(\int_{\Omega}|f(x)|^{p} d x\right)^{1 / p}<\infty, \quad 0<p<\infty, \\
\left\|f\left|L^{\infty}(\Omega) \|=\underset{x \in \Omega}{\operatorname{ess}-\sup }\right| f(x) \mid<\infty .\right.
\end{gathered}
$$

By $\mathcal{S}\left(\mathbb{R}^{n}\right)$ we denote the Schwartz space of all complex-valued, infinitely differentiable, and rapidly decreasing functions on $\mathbb{R}^{n}$ and by $\mathcal{S}^{\prime}\left(\mathbb{R}^{n}\right)$ the dual space of all tempered distributions on $\mathbb{R}^{n}$. We define the Fourier transform of a function $f \in \mathcal{S}\left(\mathbb{R}^{n}\right)$ by

$$
\mathcal{F}(f)(\xi)=(2 \pi)^{-n / 2} \int_{\mathbb{R}^{n}} e^{-i x \cdot \xi} f(x) d x
$$

Its inverse is denoted by $\mathcal{F}^{-1} f$. Both $\mathcal{F}$ and $\mathcal{F}^{-1}$ are extended to the dual Schwartz space $\mathcal{S}^{\prime}\left(\mathbb{R}^{n}\right)$ in the usual way. such that

Let $\tau \in[0, \infty)$ and $p \in(0, \infty]$. Let $L_{\tau}^{p}\left(\mathbb{R}^{n}\right)$ be the collection of functions $f \in L_{\mathrm{loc}}^{p}\left(\mathbb{R}^{n}\right)$

$$
\left\|f \mid L_{\tau}^{p}\left(\mathbb{R}^{n}\right)\right\|=\sup _{B_{J}} \frac{1}{\left|B_{J}\right|^{\tau}}\left(\int_{B_{J}}|f(x)|^{p} d x\right)^{1 / p}<\infty
$$

where the supremum is taken over all $J \in \mathbb{Z} \backslash \mathbb{N}$ and all balls $B_{J}$ of $\mathbb{R}^{n}$ with radius $2^{-J}$. Obviously, when $\tau=0$, then $L_{\tau}^{p}\left(\mathbb{R}^{n}\right)=L^{p}\left(\mathbb{R}^{n}\right)$. Furthermore,

$$
L_{\tau}^{p}\left(\mathbb{R}^{n}\right) \hookrightarrow S^{\prime}\left(\mathbb{R}^{n}\right),
$$

(see [9, page 46]). 
If $s \in \mathbb{R}, 0<q \leq \infty$ and $J \in \mathbb{Z}$, then $\ell_{q, J^{+}}^{s}$ is the set of all sequences $\left\{f_{k}\right\}_{k \geq J^{+}}$of complex numbers such that

$$
\left\|\left\{f_{k}\right\}_{k \geq J^{+}} \mid \ell_{q, J^{+}}^{s}\right\|=\left(\sum_{k \geq J^{+}} 2^{k s q}\left|f_{k}\right|^{q}\right)^{1 / q}<\infty,
$$

with the obvious modification if $q=\infty$. We recall that for any $0<\theta \leq 1$ and any $J \in \mathbb{Z}$

$$
\begin{gathered}
\left(\sum_{k \geq J^{+}}\left|f_{k}\right|\right)^{\theta} \leq \sum_{k \geq J^{+}}\left|f_{k}\right|^{\theta}, \\
(y+z)^{d} \leq \max \left(1,2^{d-1}\right)\left(y^{d}+z^{d}\right), \quad y, z \geq 0, d>0 .
\end{gathered}
$$

Let $f$ be an arbitrary function on $\mathbb{R}^{n}$ and $x, h \in \mathbb{R}^{n}$. Then

$$
\Delta_{h} f(x)=f(x+h)-f(x), \quad \Delta_{h}^{M+1} f(x)=\Delta_{h}\left(\Delta_{h}^{M} f\right)(x), \quad M \in \mathbb{N} .
$$

These are the well-known differences of functions which play an important role in the theory of function spaces. Using mathematical induction one can show the explicit formula

$$
\Delta_{h}^{M} f(x)=\sum_{j=0}^{M}(-1)^{j} C_{j}^{M} f(x+(M-j) h),
$$

where $C_{j}^{M}$ are the binomial coefficients.

Recall that $\eta_{j, N}(x)=2^{j n}\left(1+2^{j}|x|\right)^{-N}$, for any $x \in \mathbb{R}^{n}, j \in \mathbb{N}_{0}$ and $N>0$. By $c$ we denote generic positive constants, which may have different values at different occurrences.

\subsection{The $B_{p, q}^{s, \tau}$ Spaces and $F_{p, q}^{s, \tau}$ Spaces}

In this subsection we present the Fourier analytical definition of $B_{p, q}^{s, \tau}$ spaces, $F_{p, q}^{s, \tau}$ spaces and recall their basic properties. We first need the concept of a smooth dyadic resolution of unity.

Definition 2.1. Let $\Psi$ be a function in $\mathcal{S}\left(\mathbb{R}^{n}\right)$ satisfying $\Psi(x)=1$ for $|x| \leq 1$ and $\Psi(x)=0$ for $|x| \geq 3 / 2$. We put $\varphi_{0}(x)=\Psi(x), \varphi_{1}(x)=\Psi(x / 2)-\Psi(x)$ and

$$
\varphi_{j}(x)=\varphi_{1}\left(2^{-j+1} x\right) \text { for } j=2,3, \ldots
$$

Then we have supp $\varphi_{j} \subset\left\{x \in \mathbb{R}^{n}: 2^{j-1} \leq|x| \leq 3 \cdot 2^{j-1}\right\}, \varphi_{j}(x)=1$ for $3 \cdot 2^{j-2} \leq|x| \leq 2^{j}$ and $\Psi(x)+\sum_{j \geq 1} \varphi_{j}(x)=1$ for all $x \in \mathbb{R}^{n}$. The system of functions $\left\{\varphi_{j}\right\}$ is called a smooth dyadic resolution of unity. We define the convolution operators $\Delta_{j}$ by the following:

$$
\Delta_{j} f=\mathcal{F}^{-1} \varphi_{j} * f, \quad j \in \mathbb{N}, \quad \Delta_{0} f=\mathcal{F}^{-1} \Psi * f, \quad f \in \mathcal{S}^{\prime}\left(\mathbb{R}^{n}\right) .
$$


Thus we obtain the Littlewood-Paley decomposition

$$
f=\sum_{j \geq 0} \Delta_{j} f
$$

of all $f \in \mathcal{S}^{\prime}\left(\mathbb{R}^{n}\right)$ (convergence in $\mathcal{S}^{\prime}\left(\mathbb{R}^{n}\right)$ ).

The $B_{p, q}^{s, \tau}$ spaces and $F_{p, q}^{s, \tau}$ spaces are defined in the following way.

Definition 2.2. (i) Let $s \in \mathbb{R}, \tau \in[0, \infty)$ and $0<p, q \leq \infty$. The space $B_{p, q}^{s, \tau}$ is the collection of all $f \in \mathcal{S}^{\prime}\left(\mathbb{R}^{n}\right)$ such that

$$
\left\|f \mid B_{p, q}^{s, \tau}\right\|=\sup _{B_{J}} \frac{1}{\left|B_{J}\right|^{\tau}}\left(\sum_{j \geq J^{+}} 2^{j s q}\left\|\Delta_{j} f \mid L^{p}\left(B_{J}\right)\right\|^{q}\right)^{1 / q}<\infty,
$$

where the supremum is taken over all $J \in \mathbb{Z}$ and all balls $B_{J}$ of $\mathbb{R}^{n}$ with radius $2^{-J}$.

(ii) Let $s \in \mathbb{R}, \tau \in[0, \infty), 0<p<\infty$ and $0<q \leq \infty$. The space $F_{p, q}^{s, \tau}$ is the collection of all $f \in \mathcal{S}^{\prime}\left(\mathbb{R}^{n}\right)$ such that

$$
\left\|f\left|F_{p, q}^{s, \tau}\left\|=\sup _{B_{J}} \frac{1}{\left|B_{J}\right|^{\tau}}\right\|\left(\sum_{j \geq J^{+}} 2^{j s q}\left|\Delta_{j} f\right|^{q}\right)^{1 / q}\right| L^{p}\left(B_{J}\right)\right\|<\infty,
$$

where the supremum is taken over all $J \in \mathbb{Z}$ and all balls $B_{J}$ of $\mathbb{R}^{n}$ with radius $2^{-J}$.

Remark 2.3. The spaces $B_{p, q}^{s, \tau}$ and $F_{p, q}^{s, \tau}$ are independent of the particular choice of the smooth dyadic resolution of unity $\left\{\varphi_{j}\right\}$ appearing in their definitions. They are quasi-Banach spaces (Banach spaces if $p \geq 1, q \geq 1$ ). In particular,

$$
B_{p, q}^{s, 0}=B_{p, q}^{s}, \quad F_{p, q}^{s, 0}=F_{p, q}^{s}
$$

where $B_{p, q}^{s}$ and $F_{p, q}^{s}$ are the Besov spaces and Triebel-Lizorkin spaces respectively. If we replace the balls $B_{J}$ by dyadic cubes $P$ (with side length $2^{-J}$ ) we obtain equivalent norms.

The full treatment of both scales of spaces can be found in [9]. Let $\Delta_{j} f\left(j \in \mathbb{N}_{0}\right)$ be the functions introduced in Definition 2.1. For any $a>0$, any $x \in \mathbb{R}^{n}$ and any $J \in \mathbb{Z}$ we denote (Peetre's maximal functions)

$$
\Delta_{j, J}^{*, a} f(x)=\sup _{y \in B_{J}} \frac{\left|\Delta_{j} f(y)\right|}{\left(1+2^{j}|x-y|\right)^{a}} \quad j \in \mathbb{N}_{0}, f \in \mathcal{S}^{\prime}\left(\mathbb{R}^{n}\right) .
$$

We now present a fundamental characterization of $B_{p, q}^{s, \tau}$ spaces and $F_{p, q}^{s, \tau}$ spaces. 
Journal of Function Spaces and Applications

Theorem 2.4. Let $s \in \mathbb{R}, \tau \in[0, \infty), 0<p, q \leq \infty$ and $a>n / p$. Then

$$
\left\|f \mid B_{p, q}^{s, \tau}\right\|^{*}=\sup _{B_{J}} \frac{1}{\left|B_{J}\right|^{\tau}}\left(\sum_{j \geq J^{+}} 2^{j s q}\left\|\Delta_{j, J}^{*, a} f \mid L^{p}\left(B_{J}\right)\right\|^{q}\right)^{1 / q},
$$

is an equivalent quasinorm in $B_{p, q}^{s, \tau}$.

Theorem 2.5. Let $s \in \mathbb{R}, \tau \in[0, \infty), 0<p<\infty, 0<q \leq \infty$ and $a>n / \min (p, q)$. Then $\left\|f\left|F_{p, q}^{s, \tau}\left\|^{*}=\sup _{B_{J}}\left(1 /\left|B_{J}\right|^{\tau}\right)\right\|\left(\sum_{j \geq J^{+}} 2^{j s q}\left|\Delta_{j, J}^{*, a} f\right|^{q}\right)^{1 / q}\right| L^{p}\left(B_{J}\right)\right\|$, is an equivalent quasinorm in $F_{p, q}^{s, \tau}$.

Remark 2.6. Theorem 2.4 for $0<p, q<\infty$ is given in [8, Theorem 4.5]. For Theorem 2.5 see $\left[12\right.$, Theorem 1.1]. In addition if $a>n \max (1 / p, \tau)$, then in Theorem $2.4 \Delta_{j, J}^{*, a} f$ can be replaced by $\Delta_{j}^{*, a} f$, where

$$
\Delta_{j}^{*, a} f(x)=\sup _{y \in \mathbb{R}^{n}} \frac{\left|\Delta_{j} f(y)\right|}{\left(1+2^{j}|x-y|\right)^{a}} \quad j \in \mathbb{N}_{0}, f \in \mathcal{S}^{\prime}\left(\mathbb{R}^{n}\right) .
$$

\section{Some Technical Lemmas}

To prove our results, we need some technical lemmas. The following lemma for $\Delta_{j} f$, in place of $\Delta_{j}^{*, a} f$, is given in [14, pages 87-89] (for the $B_{p, q}^{s, \tau}$ spaces and $1 \leq p<\infty$ ). Further results, can be found in [12, Lemma 2.4].

Lemma 3.1. Let $\Delta_{j} f$ be as in Definition 2.1 and let $s \in \mathbb{R}, a>0, \tau \in[0, \infty)$ and $0<p, q \leq$ $\infty\left(0<p<\infty\right.$ for the space $\left.F_{p, q}^{s, \tau}\right)$. Then there is a constant $c>0$, independent of $j$, such that for any $f \in \mathcal{S}^{\prime}\left(\mathbb{R}^{n}\right)$

$$
\left\|\Delta_{j}^{*, a} f\right\|_{\infty} \leq c 2^{j(n / p-s-n \tau)}\left\|f \mid A_{p, q}^{s, \tau}\right\|, \quad j \in \mathbb{N}_{0} .
$$

Here one uses $A_{p, q}^{s, \tau}$ to denote either $B_{p, q}^{s, \tau}$ or $F_{p, q}^{s, \tau}$.

Proof. Let $\psi, \psi_{0} \in \mathcal{S}\left(\mathbb{R}^{n}\right)$ be two functions such that $\mathscr{F} \psi=1$ and $\mathcal{F} \psi_{0}=1$ on supp $\varphi_{1}$ and $\operatorname{supp} \varphi_{0}$ respectively. Then

$$
\left|\Delta_{j} f(y)\right|=\left|\psi_{j} * \Delta_{j} f(y)\right|, \quad y \in \mathbb{R}^{n}
$$

with $\psi_{j}=2^{(j-1) n} \psi\left(2^{j-1}\right)$ if $j \in \mathbb{N}$. Since $\varphi \in \mathcal{S}\left(\mathbb{R}^{n}\right)$, the right-hand side is bounded by $c \eta_{j-1, N} *$ $\left|\Delta_{j} f\right|(y)$, for any $N>0$. Hence we get for all $f \in \mathcal{S}^{\prime}\left(\mathbb{R}^{n}\right)$ and any $x \in \mathbb{R}^{n}$

$$
\Delta_{j}^{*, a} f(x) \leq c \sup _{y \in \mathbb{R}^{n}} \eta_{j-1, N} *\left|\Delta_{j} f\right|(y) .
$$


Using the same method given in [9, Proposition 2.6] we obtain for any $y \in \mathbb{R}^{n}$

$$
\eta_{j-1, N} *\left|\Delta_{j} f\right|(y) \leq c 2^{j(n / p-s-n \tau)}\left\|f \mid A_{p, q}^{s, \tau}\right\| .
$$

The proof is completed.

Lemma 3.2. Let $M \in \mathbb{N}, J \in \mathbb{Z} \backslash \mathbb{N}, A>0, \tau \in[0, \infty)$ and $1 \leq p \leq \infty$. Then there is a constant $c>0$, independent of $J$ and $A$, such that

$$
\left\|\int_{|h| \leq A}\left|\Delta_{h}^{M} f(\cdot)\right| d h\left|L^{p}\left(B_{J}\right)\left\|\leq c A^{n}\left|B_{J}\right|^{\tau}\right\| f\right| L_{\tau}^{p}\right\|,
$$

for any ball $B_{J}$ of $\mathbb{R}^{n}$ with radius $2^{-J}$ and any function $f$ such that $\left\|f \mid L_{\tau}^{p}\right\|<\infty$.

Proof. Since $1 \leq p \leq \infty$, the left-hand side is bounded by

$$
\int_{|h| \leq A}\left\|\Delta_{h}^{M} f \mid L^{p}\left(B_{J}\right)\right\| d h
$$

From the definition of $\Delta_{h}^{M} f$ we have

$$
\left|\Delta_{h}^{M} f(x)\right| \leq \sum_{m=0}^{M} C_{m}^{M}|f(x+(M-m) h)|
$$

Take the $L^{p}\left(B_{J}\right)$-norm to estimate (3.6) form above by

$$
\sum_{m=0}^{M} C_{m}^{M} \int_{|h| \leq A}\left\|f \mid L^{p}\left(\widetilde{B}_{J}\right)\right\| d h,
$$

where if $x_{0}$ the centre of $B_{J}$ then $x_{0}+(M-m) h$ is the centre of $\widetilde{B}_{J}$. Using the fact that $\left|\widetilde{B}_{J}\right|=\left|B_{J}\right|$ to estimate (3.8) from above by $c A^{n}\left|B_{J}\right|^{\tau}\left\|f \mid L_{\tau}^{p}\right\|$.

The lemma is proved.

Remark 3.3. Let $M, A, \tau$, and $p$ be as in Lemma 3.2. Let $J \in \mathbb{N}_{0}$. By the embedding $L^{p}\left(B_{0}\right) \hookrightarrow$ $L^{p}\left(B_{J}\right)$ there is a constant $c>0$, independent of $J$ and $A$, such that

$$
\left\|\int_{|h| \leq A}\left|\Delta_{h}^{M} f(\cdot)\right| d h\left|L^{p}\left(B_{J}\right)\left\|\leq c A^{n}\right\| f\right| L_{\tau}^{p}\right\|,
$$


for any ball $B_{J}$ of $\mathbb{R}^{n}$ with radius $2^{-J}$ and any function $f$ such that $\left\|f \mid L_{\tau}^{p}\right\|<\infty$.

For $s>0, M \in \mathbb{N}, \tau \in[0, \infty), 1 \leq p<\infty$ and $0<q \leq \infty$, we set

$$
\left\|f\left|F_{p, q}^{s, \tau}\left\|_{M}=\right\| f\right| L_{\tau}^{p}\left(\mathbb{R}^{n}\right)\right\|+\sup _{B_{J}} \frac{1}{\left|B_{J}\right|^{\tau}}\left\|\left(\int_{0}^{2^{-J^{+}+1}} t^{-s q} \sup _{|h| \leq t}\left|\Delta_{h}^{M} f(\cdot)\right|^{q} \frac{d t}{t}\right)^{1 / q} \mid L^{p}\left(B_{J}\right)\right\| .
$$

Here the supremum is taken over all $J \in \mathbb{Z}$ and all balls $B_{J}$ of $\mathbb{R}^{n}$ with radius $2^{-J}$.

Lemma 3.4. Let $s>0, M \in \mathbb{N}, J \in \mathbb{Z}, \tau \in[0, \infty), 1 \leq p<\infty$ and $0<q \leq \infty$. Then there is $a$ constant $c>0$, independent of $J$, such that

$$
\begin{gathered}
\left\|\left(\sum_{j \geq J^{+}} 2^{j s q}\left(\int_{|v| \leq 1}\left|\Delta_{2^{-j} v}^{M} f(\cdot)\right| d v\right)^{q}\right)^{1 / q}\left|L^{p}\left(B_{J}\right)\left\|\leq c\left|B_{J}\right|^{\tau}\right\| f\right| F_{p, q}^{s, \tau}\right\|_{M^{\prime}} \\
\left\|\left(\sum_{j \geq J^{+}} 2^{j s q}\left(\int_{|v|>1}\left|\Delta_{2^{-j} v}^{M} f(\cdot) \omega(v)\right| d v\right)^{q}\right)^{1 / q}\left|L^{p}\left(B_{J}\right)\left\|\leq c\left|B_{J}\right|^{\tau}\right\| f\right| F_{p, q}^{s, \tau}\right\|_{M^{\prime}}
\end{gathered}
$$

for any ball $B_{J}$ of $\mathbb{R}^{n}$ with radius $2^{-J}$, any $\omega \in \mathcal{S}\left(\mathbb{R}^{n}\right)$ and any function $f$ such that $\left\|f \mid F_{p, q}^{s, \tau}\right\|_{M}<\infty$.

Proof. Let $P$ be a dyadic cube with side length $2^{-J}$. This result, for $P$ in place of $B_{J}$, is already known, see [9, Lemmas 4.3,4.4]. By simple modifications of their arguments we will give another proof of (3.12). The proof is given only when $0<q<\infty$. The case $q=\infty$ is similar. Before proving this result we note that for any $x \in \mathbb{R}^{n}$ and any $i \in \mathbb{Z}$

$$
\begin{aligned}
& \left(\sum_{v \geq i} 2^{(s+n) v q}\left(\int_{|h| \leq 2^{-v}}\left|\Delta_{h}^{M} f(x)\right| d h\right)^{q}\right)^{1 / q} \\
& \quad \leq c\left(\int_{0}^{2^{-i+1}} t^{-(s+n) q}\left(\int_{|h| \leq t}\left|\Delta_{h}^{M} f(x)\right| d h\right)^{q} \frac{d t}{t}\right)^{1 / q} .
\end{aligned}
$$

Here we will prove that the left-hand side of (3.12) is bounded by

$$
\left\|f\left|L_{\tau}^{p}\left(\mathbb{R}^{n}\right)\left\|+\sup _{B_{J}} \frac{1}{\left|B_{J}\right|^{\tau}}\right\|\left(\int_{0}^{2^{-J^{+}}} t^{-s q} \sup _{|h| \leq t}\left|\Delta_{h}^{M} f(\cdot)\right|^{q} \frac{d t}{t}\right)^{1 / q}\right| L^{p}\left(B_{J}\right)\right\|=\left\|f \mid F_{p, q}^{s, \tau}\right\|_{M}^{\prime} .
$$


Obviously, $\left\|f\left|F_{p, q}^{s, \tau}\left\|_{M}^{\prime} \leq\right\| f\right| F_{p, q}^{s, \tau}\right\|_{M}$. We write

$$
\begin{aligned}
2^{j s} \int_{|v|>1}\left|\Delta_{2^{-j} v}^{M} f(x) \omega(v)\right| d v & =\sum_{k=0}^{\infty} 2^{j s} \int_{2^{k}<|v| \leq 2^{k+1}}\left|\Delta_{2^{-j} v}^{M} f(x) \omega(v)\right| d v \\
& \leq c \sum_{k=0}^{\infty} 2^{(s+n) j-N k} \int_{2^{k-j}<|h| \leq 2^{k-j+1}}\left|\Delta_{h}^{M} f(x)\right| d h,
\end{aligned}
$$

where $N>0$ is at our disposal and we have used the properties of the function $\omega$,

$$
|\omega(x)| \leq c(1+|x|)^{-N},
$$

for any $x \in \mathbb{R}^{n}$ and any $N>0$. Now the right-hand side of (3.15) in $\ell_{q, J^{+}}^{s}$-norm is bounded by (with $\sigma=\min (1, q)$ )

$$
\begin{gathered}
c\left(\sum_{k=0}^{\infty} 2^{-N \sigma k}\left(\sum_{j \geq J^{+}} 2^{(s+n) j q}\left(\int_{2^{k-j}<|h| \leq 2^{k-j+1}}\left|\Delta_{h}^{M} f(x)\right| d h\right)^{q}\right)^{\sigma / q}\right)^{1 / \sigma} \\
\quad=\left(\sum_{k=0}^{J^{+}-1} \cdots+\sum_{k \geq J^{+}} \cdots\right)^{1 / \sigma}=\left(I_{J}(x)+I I_{J}(x)\right)^{1 / \sigma} \\
\leq 2^{1 / \sigma-1}\left(\left(I_{J}(x)\right)^{1 / \sigma}+\left(I I_{J}(x)\right)^{1 / \sigma}\right)
\end{gathered}
$$

by (2.7). Here we put $\sum_{k=0}^{J^{+}-1} \cdots=0$ if $J^{+}=0$. Take the $L^{p}\left(B_{J}\right)$-norm we obtain that the left-hand side of (3.12) is bounded by

$$
2^{1 / \sigma-1}\left\|\left(I_{J}\right)^{1 / \sigma}\left|L^{p}\left(B_{J}\right)\left\|+2^{1 / \sigma-1}\right\|\left(I I_{J}\right)^{1 / \sigma}\right| L^{p}\left(B_{J}\right)\right\|
$$

Let us estimate $\left(I_{J}\right)^{1 / \sigma}$ in $L^{p}\left(B_{J}\right)$-norm. After a change of variable $j-k-1=v$, we get for any $x \in \mathbb{R}^{n}$ (here $J^{+}=J$ and $k<J$ )

$$
\begin{aligned}
I_{J}(x) & \leq c \sum_{k=0}^{J^{+}-1} 2^{(s+n-N) \sigma k}\left(\sum_{v \geq J^{+}-k-1} 2^{(s+n) v q}\left(\int_{|h| \leq 2^{-v}}\left|\Delta_{h}^{M} f(x)\right| d h\right)^{q}\right)^{\sigma / q} \\
& =\sum_{k=0}^{J^{+}-2} \cdots+\sum_{k=J^{+}-1}^{J^{+}-1} \cdots \\
& =M_{1}(x)+M_{2}(x) .
\end{aligned}
$$


Journal of Function Spaces and Applications

Here we put $\sum_{k=0}^{J^{+}-2} \cdots=0$ if $J^{+} \leq 1$. We have

$$
M_{1}(x) \leq c \sum_{k=0}^{J^{+}-2} 2^{(s+n-N) \sigma k}\left(\int_{0}^{2^{-J+k+2}} t^{-(s+n) q}\left(\int_{|h| \leq t}\left|\Delta_{h}^{M} f(x)\right| d h\right)^{q} \frac{d t}{t}\right)^{\sigma / q} .
$$

Since $L^{p / \sigma}\left(B_{J}\right)$ is a normed spaces and $B_{J} \subset B_{J-k-2}$, the right-hand side in $L^{p / \sigma}\left(B_{J}\right)$-norm can be estimated from above by

$$
\begin{aligned}
& c \sum_{k=0}^{J^{+}-2} 2^{(s+n-N) \sigma k}\left\|\left(\int_{0}^{2^{-J+k+2}} t_{|h| \leq t}^{-s q} \sup _{h}\left|\Delta_{h}^{M} f(\cdot)\right|^{q} \frac{d t}{t}\right)^{1 / q} \mid L^{p}\left(B_{J-k-2}\right)\right\|^{\sigma} \\
& \leq c\left|B_{J}\right|^{\tau \sigma} \sum_{k=0}^{J^{+}-2} 2^{(s+n-N+n \tau) \sigma k}\left(\left\|f \mid F_{p, q}^{s, \tau}\right\|_{M}^{\prime}\right)^{\sigma} .
\end{aligned}
$$

We choose $N>2(s+n)+\tau n$. This yields that the last expression is bounded by

$$
c\left|B_{J}\right|^{\tau \sigma}\left(\left\|f \mid F_{p, q}^{s, \tau}\right\|_{M}^{\prime}\right)^{\sigma}
$$

where $c>0$ is independent of $J$. Now $\left(M_{2}\right)^{1 / \sigma}$ in $L^{p}\left(B_{J}\right)$-norm is bounded by

$$
\begin{gathered}
c 2^{(s+n-N) J^{+}}\left\|\left(\sum_{v \geq 0} 2^{(s+n) v q}\left(\int_{|h| \leq 2^{-v}}\left|\Delta_{h}^{M} f(\cdot)\right| d h\right)^{q}\right)^{1 / q} \mid L^{p}\left(B_{J}\right)\right\| \\
\leq c 2^{(s+n-N) J^{+}}\left\|\left(\sum_{v \geq 1} 2^{(s+n) v q}\left(\int_{|h| \leq 2^{-v}}\left|\Delta_{h}^{M} f(\cdot)\right| d h\right)^{q}\right)^{1 / q} \mid L^{p}\left(B_{J}\right)\right\| \\
+c 2^{(s+n-N) J^{+}}\left\|\int_{|h| \leq 1}\left|\Delta_{h}^{M} f(\cdot)\right| d h \mid L^{p}\left(B_{J}\right)\right\|,
\end{gathered}
$$

where we have used (2.7). Using the embedding $L^{p}\left(B_{0}\right) \hookrightarrow L^{p}\left(B_{J}\right)$ and Remark 3.3, we obtain

$$
\begin{aligned}
\left\|M_{2} \mid L^{p / \sigma}\left(B_{J}\right)\right\| & =\left\|\left(M_{2}\right)^{1 / \sigma} \mid L^{p}\left(B_{J}\right)\right\|^{\sigma} \\
& \leq c 2^{(s+n-N) \sigma J^{+}}\left(\left\|f \mid F_{p, q}^{s, \tau}\right\|_{M}^{\prime}\right)^{\sigma} \\
& =c 2^{(s+n-N+n \tau) \sigma J^{+}}\left|B_{J}\right|^{\tau \sigma}\left(\left\|f \mid F_{p, q}^{s, \tau}\right\|_{M}^{\prime}\right)^{\sigma} \\
& \leq c\left|B_{J}\right|^{\tau \sigma}\left(\left\|f \mid F_{p, q}^{s, \tau}\right\|_{M}^{\prime}\right)^{\sigma}
\end{aligned}
$$


because of $N>s+n+n \tau$. Therefore,

$$
\begin{aligned}
\left\|\left(I_{J}\right)^{1 / \sigma} \mid L^{p}\left(B_{J}\right)\right\|^{\sigma} & =\left\|I_{J} \mid L^{p / \sigma}\left(B_{J}\right)\right\| \\
& \leq\left\|M_{1}\left|L^{p / \sigma}\left(B_{J}\right)\|+\| M_{2}\right| L^{p / \sigma}\left(B_{J}\right)\right\| \\
& \leq c\left|B_{J}\right|^{\tau \sigma}\left(\left\|f \mid F_{p, q}^{s, \tau}\right\|_{M}^{\prime}\right)^{\sigma} .
\end{aligned}
$$

Now let us estimate $\left(I I_{J}\right)^{1 / \sigma}$ in $L^{p}\left(B_{J}\right)$-norm. We write

$$
\begin{aligned}
I I_{J}(\cdot) & =c \sum_{k \geq J^{+}} 2^{-N \sigma k}\left(\sum_{j=J^{+}}^{k+J^{+}+1} \cdots+\sum_{j=k+J^{+}+2}^{\infty} \cdots\right)^{\sigma / q} \\
& \leq c \sum_{k \geq J^{+}} 2^{-N \sigma k}\left(\left(\sum_{j=J^{+}}^{k+J^{+}+1} \cdots\right)^{\sigma / q}+\left(\sum_{j=k+J^{+}+2}^{\infty} \cdots\right)^{\sigma / q}\right) \\
& =c \sum_{k \geq J^{+}} 2^{-N \sigma k}\left(S_{k}^{1}(\cdot)+S_{k}^{2}(\cdot)\right) .
\end{aligned}
$$

After a change of variable $j-k-1=v$, we get

$$
\begin{aligned}
\left(S_{k}^{1}(x)\right)^{1 / \sigma} & \leq c 2^{(s+n) k}\left(\sum_{v=J^{+}-k-1}^{J^{+}} 2^{(s+n) v q}\left(\int_{|h| \leq 2^{-v}}\left|\Delta_{h}^{M} f(x)\right| d h\right)^{q}\right)^{1 / q} \\
& \leq c 2^{(s+n) k}\left(\int_{2^{-J^{+}}}^{2^{k-J^{+}+2}} t^{-(s+n) q}\left(\int_{|h| \leq t}\left|\Delta_{h}^{M} f(x)\right| d h\right)^{q} \frac{d t}{t}\right)^{1 / q} \\
& \leq c 2^{(s+n) k} \int_{|h| \leq 2^{k-J^{+}+2}}\left|\Delta_{h}^{M} f(x)\right| d h\left(\int_{2^{-J^{+}}}^{2^{k-J^{+}+2}} t^{-(s+n) q} \frac{d t}{t}\right)^{1 / q} \\
& \leq c 2^{(s+n) J^{+}+(s+n) k} \int_{|h| \leq 2^{k-J^{+}+2}}\left|\Delta_{h}^{M} f(x)\right| d h .
\end{aligned}
$$

Therefore there exists a constant $c>0$ independent of $J$ and $k$ such that

$$
\begin{aligned}
\left\|\left(S_{k}^{1}\right)^{1 / \sigma} \mid L^{p}\left(B_{J}\right)\right\| & \leq c 2^{(s+n) J^{+}+(s+n) k}\left\|\int_{|h| \leq 2^{k-J^{+}+2}} \Delta_{h}^{M} f(\cdot) d h \mid L^{p}\left(B_{J}\right)\right\| \\
& \leq c 2^{(s+\tau n) J^{+}+(s+2 n) k}\left|B_{J}\right|^{\tau}\left\|f \mid L_{\tau}^{p}\right\| \\
& \leq c 2^{(2 s+2 n+\tau n) k}\left|B_{J}\right|^{\tau}\left\|f \mid F_{p, q}^{s, \tau}\right\|_{M^{\prime}}^{\prime}
\end{aligned}
$$


Journal of Function Spaces and Applications

by Lemma 3.2 (combined with Remark 3.3) and the fact that $k \geq J^{+}$. Now let us estimate $\left(S_{k}^{2}\right)^{1 / \sigma}$ in $L^{p}\left(B_{J}\right)$-norm. We have

$$
\begin{aligned}
\left(S_{k}^{2}(x)\right)^{1 / \sigma} & \leq c 2^{(n+s) k}\left(\sum_{v=J^{+}+1}^{\infty} 2^{(s+n) v q}\left(\int_{|h| \leq 2^{-v}}\left|\Delta_{h}^{M} f(x)\right| d h\right)^{q}\right)^{1 / q} \\
& \leq c 2^{(n+s) k}\left(\int_{0}^{2^{-J^{+}}} t^{-s q} \sup _{|h| \leq t}\left|\Delta_{h}^{M} f(x)\right|^{q} \frac{d t}{t}\right)^{1 / q}
\end{aligned}
$$

Therefore,

$$
\begin{aligned}
\left\|\left(S_{k}^{2}\right)^{1 / \sigma} \mid L^{p}\left(B_{J}\right)\right\| & \leq c 2^{(n+s) k}\left\|\left(\int_{0}^{2^{-J^{+}}} t_{|h| \leq t}^{-s q} \sup _{h}\left|\Delta_{h}^{M} f(\cdot)\right|^{q} \frac{d t}{t}\right)^{1 / q} \mid L^{p}\left(B_{J}\right)\right\| \\
& \leq c 2^{(n+s) k}\left|B_{J}\right|^{\tau}\left\|f \mid F_{p, q}^{s, \tau}\right\|_{M}^{\prime} .
\end{aligned}
$$

Consequently,

$$
\begin{aligned}
\left\|\left(I I_{J}\right)^{1 / \sigma} \mid L^{p}\left(B_{J}\right)\right\|^{\sigma} & =\left\|I I_{J} \mid L^{p / \sigma}\left(B_{J}\right)\right\| \\
& \leq \sum_{k \geq J^{+}} 2^{-N \sigma k}\left(\left\|\left(S_{k}^{1}\right)^{1 / \sigma}\left|L^{p}\left(B_{J}\right)\left\|^{\sigma}+\right\|\left(S_{k}^{2}\right)^{1 / \sigma}\right| L^{p}\left(B_{J}\right)\right\|^{\sigma}\right) \\
& \leq c \sum_{k \geq J^{+}} 2^{(2 s+2 n+\tau n-N) \sigma k}\left|B_{J}\right|^{\tau \sigma}\left(\left\|f \mid F_{p, q}^{s, \tau}\right\|_{M}^{\prime}\right)^{\sigma} \\
& \leq c\left|B_{J}\right|^{\tau \sigma}\left(\left\|f \mid F_{p, q}^{s, \tau}\right\|_{M}^{\prime}\right)^{\sigma},
\end{aligned}
$$

since $N>2(s+n)+\tau n$. This finishes the proof of Lemma 3.4.

For $s>0, M \in \mathbb{N}, \tau \in[0, \infty), 1 \leq p \leq \infty$, and $0<q \leq \infty$, we set

$$
\left\|f\left|B_{p, q}^{s, \tau}\left\|_{M}=\right\| f\right| L_{\tau}^{p}\left(\mathbb{R}^{n}\right)\right\|+\sup _{B_{J}} \frac{1}{\left|B_{J}\right|^{\tau}}\left(\int_{0}^{2^{-J^{+}+1}} t^{-s q} \sup _{|h| \leq t}\left\|\Delta_{h}^{M} f \mid L^{p}\left(B_{J}\right)\right\|^{q} \frac{d t}{t}\right)^{1 / q} .
$$

Here the supremum is taken over all $J \in \mathbb{Z}$ and all balls $B_{J}$ of $\mathbb{R}^{n}$ with radius $2^{-J}$. Similar arguments yield. 
Lemma 3.5. Let $s>0, M \in \mathbb{N}, J \in \mathbb{Z}, \tau \in[0, \infty), 1 \leq p \leq \infty$ and $0<q \leq \infty$. Then there is $a$ constant $c>0$, independent of $J$, such that

$$
\begin{gathered}
\left(\sum_{j \geq J^{+}} 2^{j s q}\left(\int_{|v| \leq 1}\left\|\Delta_{2^{-j} v}^{M} f \mid L^{p}\left(B_{J}\right)\right\| d v\right)^{q}\right)^{1 / q} \leq c\left|B_{J}\right|^{\tau}\left\|f \mid B_{p^{\prime}, q}^{s, \tau}\right\|_{M^{\prime}} \\
\left(\sum_{j \geq J^{+}} 2^{j s q}\left(\int_{|v|>1}\left\|\Delta_{2^{-j} v}^{M} f\left|L^{p}\left(B_{J}\right) \|\right| \omega(v) \mid d v\right)^{q}\right)^{1 / q} \leq c\left|B_{J}\right|^{\tau}\left\|f \mid B_{p, q}^{s, \tau}\right\|_{M^{\prime}}\right.
\end{gathered}
$$

for any ball $B_{J}$ of $\mathbb{R}^{n}$ with radius $2^{-J}$, any $\omega \in \mathcal{S}\left(\mathbb{R}^{n}\right)$ and any function $f$ such that $\left\|f \mid B_{p, q}^{s, \tau}\right\|_{M}<\infty$.

Now we recall the following lemma which is useful for us.

Lemma 3.6. Let $0<a<1, J \in \mathbb{Z}$ and $0<q \leq \infty$. Let $\left\{\varepsilon_{k}\right\}$ be a sequences of positive real numbers, such that

$$
\left\|\left\{\varepsilon_{k}\right\}_{k \geq J^{+}} \mid \ell_{q, J^{+}}^{0}\right\|=I<\infty .
$$

The sequences $\left\{\delta_{k}: \delta_{k}=\sum_{j=J^{+}}^{k} a^{k-j} \varepsilon_{j}\right\}_{k \geq J^{+}}$and $\left\{\eta_{k}: \eta_{k}=\sum_{j=k}^{\infty} a^{j-k} \varepsilon_{j}\right\}_{k \geq J^{+}}$are in $\ell_{q, J^{+}}^{0}$ with

$$
\left\|\left\{\delta_{k}\right\}_{k \geq J^{+}}\left|\ell_{q, J^{+}}^{0}\|+\|\left\{\eta_{k}\right\}_{k \geq J^{+}}\right| \ell_{q, J^{+}}^{0}\right\| \leq c I,
$$

$c$ depends only on $a$ and $q$.

\section{Characterizations with Differences}

We are able to state the main results of this paper.

Theorem 4.1. Let $1 \leq p<\infty, 0<q \leq \infty, \tau \in[0, \infty)$ and $M \in \mathbb{N}$. Assume

$$
\frac{n}{\min (p, q)}<s<M, \quad 0 \leq \tau<\frac{1}{p}
$$

or

$$
\frac{n}{\min (p, q)}<s<M-n \tau+\frac{n}{p^{\prime}} \quad \tau \geq \frac{1}{p}
$$

Then $\left\|f \mid F_{p, q}^{s, \tau}\right\|_{M}$ is an equivalent quasinorm in $F_{p, q}^{s, \tau}$.

Theorem 4.2. Let $1 \leq p \leq \infty, 0<q \leq \infty, \tau \in[0, \infty)$ and $M \in \mathbb{N}$. Assume

$$
0<s<M, \quad 0 \leq \tau<\frac{1}{p}
$$

or

$$
0<s<M-n \tau+\frac{n}{p}, \quad \tau \geq \frac{1}{p} .
$$

Then $\left\|f \mid B_{p, q}^{s, \tau}\right\|_{M}$ is an equivalent quasinorm in $B_{p, q}^{s, \tau}$. 
Journal of Function Spaces and Applications

Remark 4.3. Theorems 4.1 and 4.2 for $0 \leq \tau<s / n+1 / p$ and $0 \leq \tau<s / n+1 / p-$ $\max (1 / \min (p, q)-1,0)$, respectively, are given in [9] Theorems 4.6 and 4.7 , respectively.

Proof of Theorem 4.1. Let $B_{J}$ be any ball centered at $x_{0} \in \mathbb{R}^{n}$ and of radius $2^{-J}, J \in \mathbb{Z}$. We will do the proof in three steps.

Step 1. We have with $s>0$,

$$
\begin{aligned}
\sum_{j \geq 0}\left|\Delta_{j} f\right| & =\sum_{j \geq 0} 2^{-j s} 2^{j s}\left|\Delta_{j} f\right| \leq c \sup _{j \in \mathbb{N}_{0}} 2^{j s}\left|\Delta_{j} f\right| \\
& \leq c\left(\sum_{j \geq 0} 2^{j s q}\left|\Delta_{j} f\right|^{q}\right)^{1 / q} .
\end{aligned}
$$

Let $f \in F_{p, q}^{s, \tau}$. Then,

$$
\begin{aligned}
\left\|f \mid L_{\tau}^{p}\left(\mathbb{R}^{n}\right)\right\| & \leq\left\|\sum_{j \geq 0}\left|\Delta_{j} f\right|\left|L_{\tau}^{p}\left(\mathbb{R}^{n}\right)\|\leq c\|\left(\sum_{j \geq 0} 2^{j s q}\left|\Delta_{j} f\right|^{q}\right)^{1 / q}\right| L_{\tau}^{p}\left(\mathbb{R}^{n}\right)\right\| \\
& \leq c\left\|f \mid F_{p, q}^{s, \tau}\right\| .
\end{aligned}
$$

Step 2. For any $x \in B_{J}$ we put

$$
H_{J}(x)=\int_{0}^{2^{-J^{+}+1}} t^{-s q} \sup _{|h| \leq t}\left|\Delta_{h}^{M} f(x)\right|^{q} \frac{d t}{t}
$$

After a change of variable $t=2^{-y}$, we get

$$
H_{J}(x)=\ln 2 \int_{J^{+-1}}^{+\infty} 2^{y s q} \sup _{|h| \leq 2^{-y}}\left|\Delta_{h}^{M} f(x)\right|^{q} d y
$$

Then

$$
H_{J}(x) \leq c \sum_{k \geq J^{+}} 2^{k s q} \sup _{|h| \leq 2^{-k+1}}\left|\Delta_{h}^{M} f(x)\right|^{q}
$$

Let $\psi, \psi_{0} \in \mathcal{S}\left(\mathbb{R}^{n}\right)$ be two functions such that $\mathcal{F} \psi=1$ and $\mathcal{F} \psi_{0}=1$ on $\operatorname{supp} \varphi_{1}$ and $\operatorname{supp} \Psi$ respectively. Using the mean value theorem we obtain for any $x \in B_{J}, j \in \mathbb{N}_{0}$, and $|h| \leq 2^{-k+1}$

$$
\begin{aligned}
\left|\Delta_{h}^{1} \Delta_{j} f(x)\right| & =\left|\Delta_{h}^{1}\left(\psi_{j} * \Delta_{j} f\right)(x)\right| \\
& \leq 2^{-k} \sup _{|x-y| \leq c 2^{-k}|\alpha|=1}\left|D^{\alpha} \psi_{j} * \Delta_{j} f(y)\right|,
\end{aligned}
$$


with some positive constant $c$, independent of $j$ and $k$, and $\psi_{j}(\cdot)=2^{(j-1) n} \psi\left(2^{j-1} \cdot\right)$ for $j=$ $1,2, \ldots$. By induction on $M$, we show that

$$
\left|\Delta_{h}^{M} \Delta_{j} f(x)\right| \leq 2^{-k M} \sup _{|x-y| \leq c 2^{-k}} \sum_{|\alpha|=M}\left|D^{\alpha} \psi_{j} * \Delta_{j} f(y)\right|
$$

We see that if $|\alpha|=M$ and $a>0$

$$
\begin{aligned}
\left|D^{\alpha} \psi_{j} * \Delta_{j} f(y)\right| & =2^{(j-1) n}\left|\int_{\mathbb{R}^{n}} D^{\alpha}\left(\psi\left(2^{j-1}(y-z)\right)\right) \Delta_{j} f(z) d z\right| \\
& \leq 2^{(j-1)(n+M)} \int_{\mathbb{R}^{n}}\left|\left(D^{\alpha} \psi\right)\left(2^{j-1}(y-z)\right)\right|\left|\Delta_{j} f(z)\right| d z .
\end{aligned}
$$

Suppose that $0 \leq j \leq J^{+}-1$. The right-hand side in (4.12) may be estimated as follows:

$$
\begin{aligned}
& c 2^{j(n+M)} \Delta_{j}^{*, a} f(y) \int_{\mathbb{R}^{n}}\left|\left(D^{\alpha} \psi\right)\left(2^{j-1}(y-z)\right)\right|\left(1+2^{j}|y-z|\right)^{a} d z \\
& \leq c 2^{j M} \Delta_{j}^{*, a} f(y) .
\end{aligned}
$$

Then we obtain for any $x \in B_{J},|h| \leq 2^{-k+1}$ and any $k \geq J^{+}$

$$
\begin{aligned}
\left|\Delta_{h}^{M} \Delta_{j} f(x)\right| & \leq c 2^{(j-k) M} \sup _{|x-y| \leq c 2^{-k}} \Delta_{j}^{*, a} f(y) \\
& \leq c 2^{(j-k) M}\left(1+2^{j-k}\right)^{a} \sup _{|x-y| \leq c 2^{-k}} \frac{\Delta_{j}^{* a} f(y)}{\left(1+2^{j}|x-y|\right)^{a}} \\
& \leq c 2^{(j-k) M} \Delta_{j}^{*, a} f(x),
\end{aligned}
$$

if $0 \leq j \leq J^{+}-1$.

Suppose now that $J^{+} \leq j \leq k$. By our assumption on $x$ and $k$ we have

$$
\left|y-x_{0}\right| \leq\left|x-x_{0}\right|+|x-y|<2^{-J}+c 2^{-k} \leq(c+1) 2^{-J},
$$

which implies that $y$ is located in some ball $\widetilde{B}_{J}$, where $\widetilde{B}_{J}=\left\{y \in \mathbb{R}^{n}:\left|y-x_{0}\right|<(c+1) 2^{-J}\right\}$. Writing the integral in (4.12) as follows

$$
\int_{\widetilde{B}_{J-1}} \cdots d z+\sum_{i \geq 0} \int_{\widetilde{B}_{J-i-2} \backslash \widetilde{B}_{J-i-1}} \cdots d z=I_{j, J}(y)+\sum_{i \geq 0} I I_{j, J-i}(y) .
$$

We recall that

$$
\Delta_{j, l}^{*, a} f(y)=\sup _{z \in \tilde{B}_{l}} \frac{\left|\Delta_{j} f(z)\right|}{\left(1+2^{j}|y-z|\right)^{a}}
$$


Journal of Function Spaces and Applications

for any $j \in \mathbb{N}_{0}, f \in \mathcal{S}^{\prime}\left(\mathbb{R}^{n}\right)$ and any $l \in \mathbb{Z}$. We have

$$
\begin{aligned}
I_{j, J}(y) & \leq \Delta_{j, J-1}^{*, a} f(y) \int_{\tilde{B}_{J-1}}\left|\left(D^{\alpha} \psi\right)\left(2^{j-1}(y-z)\right)\right|\left(1+2^{j}|y-z|\right)^{a} d z \\
& \leq c 2^{-j n} \Delta_{j, J-1}^{*, a} f(y) \leq c 2^{-j n} \Delta_{j, J-2}^{*, a} f(y) .
\end{aligned}
$$

Let us estimate $I I_{j, J-i}$. Since $\psi \in \mathcal{S}\left(\mathbb{R}^{n}\right)$, we have

$$
\left|D^{\alpha} \psi(x)\right| \leq c(1+|x|)^{-2 N}
$$

for any $x \in \mathbb{R}^{n}$ and any $N>0$. Then for any $N$ large enough, $I I_{j, J-i}(y)$ does not exceed

$$
\begin{aligned}
& c \Delta_{j, J-i-2}^{*, a} f(y) \int_{\tilde{B}_{J-i-2} \mid \tilde{B}_{J-i-1}}\left(1+2^{j-1}|y-z|\right)^{-2 N+a} d z \\
& \quad \leq c 2^{-i N} \Delta_{j, J-i-2}^{* a} f(y) \int_{\mathbb{R}^{n}}\left(1+2^{j-1}|y-z|\right)^{-N+a} d z \\
& \quad \leq c 2^{-i N-j n} \Delta_{j, J-i-2}^{* a} f(y),
\end{aligned}
$$

where we have used $2^{j-1}|y-z|>(c+1) 2^{j-J+i-1} \geq(c+1) 2^{i-1}$. Therefore,

$$
\left|D^{\alpha} \psi_{j} * \Delta_{j} f(y)\right| \leq c 2^{j M} \sum_{i \geq 0} 2^{-i N} \Delta_{j, J-i-2}^{*, a} f(y) .
$$

Then we obtain for any $x \in B_{J}$ any $|h| \leq 2^{-k+1}$ and any $J^{+} \leq j \leq k$

$$
\begin{aligned}
\left|\Delta_{h}^{M} \Delta_{j} f(x)\right| & \leq c 2^{(j-k) M} \sum_{i \geq 0} 2^{-i N} \sup _{|x-y| \leq c 2^{2-k}} \Delta_{j, J-i-2}^{*, a} f(y) \\
& \leq c 2^{(j-k) M}\left(1+2^{j-k}\right)^{a} \sum_{i \geq 0} 2^{-i N} \sup _{|x-y| \leq c 2^{-k}} \frac{\Delta_{j, J-i-2}^{*, a} f(y)}{\left(1+2^{j}|x-y|\right)^{a}} .
\end{aligned}
$$

Consequently, for any $J^{+} \leq j \leq k$ there is a constant $c>0$ independent of $J, j$, and $k$ such that

$$
\left|\Delta_{h}^{M} \Delta_{j} f(x)\right| \leq c 2^{(j-k) M} \sum_{i \geq 0} 2^{-i N} \Delta_{j, J-i-2}^{*, a} f(x)
$$


Finally for $j \geq k+1$ we have for $x \in B_{J}$ and $|h| \leq 2^{-k+1}$

$$
\begin{aligned}
\left|\Delta_{h}^{M} \Delta_{j} f(x)\right| & \leq \sum_{m=0}^{M} C_{m}^{M}\left|\Delta_{j} f(x+(M-m) h)\right| \\
& \leq 2^{M} \sup _{|x-y| \leq C 2^{-k}}\left|\Delta_{j} f(y)\right| \\
& \leq 2^{M} \sup _{|x-y| \leq C 2^{-k}} \frac{\left|\Delta_{j} f(y)\right|}{\left(1+2^{j}|x-y|\right)^{a}}\left(1+2^{j}|x-y|\right)^{a} .
\end{aligned}
$$

We remark also that by our assumption on $x$ and $k$ we have

$$
\left|y-x_{0}\right| \leq\left|x-x_{0}\right|+|x-y|<2^{-J}+C 2^{-k} \leq(C+1) 2^{-J},
$$

and this implies that $y$ is located in some ball $\widetilde{B}_{J}$, where $\widetilde{B}_{J}=\left\{y \in \mathbb{R}^{n}:\left|y-x_{0}\right|<(C+1) 2^{-J}\right\}$. Then,

$$
\left|\Delta_{h}^{M} \Delta_{j} f(x)\right| \leq c 2^{(j-k) a} \Delta_{j, J}^{*, a} f(x)
$$

if $j \geq k+1$, where $\Delta_{j, J}^{*, a} f$ is given in (4.17) (with $\widetilde{B}_{J}$ a ball centered at $x_{0}$ and of radius $(C+1) 2^{-J}$ ). We write,

$$
\begin{aligned}
\Delta_{h}^{M} f(x) & =\sum_{j \geq 0} \Delta_{h}^{M} \Delta_{j} f(x) \\
& =\sum_{j=0}^{J^{+}-1} \cdots+\sum_{j=J^{+}}^{k} \cdots+\sum_{j \geq k+1} \cdots
\end{aligned}
$$

Here we put $\sum_{j=0}^{J^{+}-1} \cdots=0$ if $J^{+}=0$. Let us estimate each term in $\ell_{q, J^{+}}^{s}$-norm. We have by (4.14) and Lemma 3.1

$$
\begin{aligned}
\sum_{j=0}^{J^{+}-1}\left|\Delta_{h}^{M} \Delta_{j} f(x)\right| & \leq \sum_{j=0}^{J^{+}-1} 2^{(j-k) M} \Delta_{j}^{*, a} f(x) \\
& \leq c \sum_{j=0}^{J^{+}-1} 2^{(j-k) M+j(n / p-s-n \tau)}\left\|f \mid F_{p, q}^{s, \tau}\right\| \\
& \leq c 2^{-k M} \sum_{j=0}^{J^{+}-1} 2^{j(M+n / p-s-n \tau)}\left\|f \mid F_{p, q}^{s, \tau}\right\| \\
& \leq c 2^{J(M+n / p-s-n \tau)-k M}\left\|f \mid F_{p, q}^{s, \tau}\right\|,
\end{aligned}
$$


Journal of Function Spaces and Applications

where the last inequality can be obtained by our assumption on $s$ and $\tau$. The last expression in $\ell_{q, J^{+}}^{s}$-norm does not exceed

$$
c 2^{J n(1 / p-\tau)}\left\|\left\{2^{\left(k-J^{+}\right)(s-M)}\right\}_{k \geq J^{+}}\left|\ell_{q, J^{+}}^{0}\|\| f\right| F_{p, q}^{S, \tau}\right\| \leq c 2^{J n(1 / p-\tau)}\left\|f \mid F_{p, q}^{S, \tau}\right\|,
$$

since $s<M$. Therefore,

$$
\begin{aligned}
\left(H_{J}(x)\right)^{1 / q} \leq & c 2^{J n(1 / p-\tau)}\left\|f \mid F_{p, q}^{s, \tau}\right\| \\
& +c\left(\sum_{k \geq J^{+}}\left(\sum_{i \geq 0} \sum_{j=J^{+}}^{k} 2^{(j-k)(M-s)+s j-i N}\left|\Delta_{j, J-i-2}^{*, a} f(x)\right|\right)^{q}\right)^{1 / q} \\
& +c\left(\sum_{k \geq J^{+}}\left(\sum_{j \geq k} 2^{(j-k)(a-s)+s j}\left|\Delta_{j, J}^{*, a} f(x)\right|\right)^{q}\right)^{1 / q} \cdot
\end{aligned}
$$

The second term can be estimated by (with $\sigma=\min (1, q)$ )

$$
c\left(\sum_{i \geq 0} 2^{-i N \sigma}\left(\sum_{k \geq J^{+}}\left(\sum_{j=J^{+}}^{k} 2^{(j-k)(M-s)+s j}\left|\Delta_{j, J-i-2}^{*, a} f(x)\right|\right)^{q}\right)^{\sigma / q}\right)^{1 / \sigma} .
$$

Since again $s<M$, then we can apply Lemma 3.6 to estimate the last expression by

$$
c\left(\sum_{i \geq 0} 2^{-i N \sigma}\left(\sum_{j \geq J^{+}} 2^{j s q}\left|\Delta_{j, J-i-2}^{*, a} f(x)\right|^{q}\right)^{\sigma / q}\right)^{1 / \sigma} .
$$

Since $L^{p / \sigma}\left(B_{J}\right)$ is a normed space, so $(4.31)$ in $L^{p}\left(B_{J}\right)$-norm is dominated by

$$
\begin{gathered}
c\left(\sum_{i \geq 0} 2^{-i N \sigma}\left\|\left(\sum_{j \geq J^{+}} 2^{j s q}\left|\Delta_{j, J-i-2}^{*, a} f\right|^{q}\right)^{\sigma / q} \mid L^{p / \sigma}\left(B_{J}\right)\right\|\right)^{1 / \sigma} \\
=c\left(\sum_{i \geq 0} 2^{-i N \sigma}\left\|\left(\sum_{j \geq J^{+}} 2^{j s q}\left|\Delta_{j, J-i-2}^{*, a} f\right|^{q}\right)^{1 / q} \mid L^{p}\left(B_{J}\right)\right\|\right)^{1 / \sigma} .
\end{gathered}
$$


Using the embedding $L^{p}\left(\widetilde{B}_{J-i-2}\right) \subset L^{p}\left(B_{J}\right)$ and the fact that $J^{+} \geq(J-i-2)^{+}$to estimate this expression from above by

$$
\begin{aligned}
& \left.c\left(\sum_{i \geq 0} 2^{-i N \sigma}\left\|\left(\sum_{j \geq(J-i-2)^{+}} 2^{j s q}\left|\Delta_{j, J-i-2}^{*, a} f\right|^{q}\right)^{1 / q} \mid L^{p}\left(\widetilde{B}_{J-i-2}\right)\right\|\right)^{\sigma}\right)^{1 / \sigma} \\
& \leq c\left|B_{J}\right|^{\tau}\left(\sum_{i \geq 0} 2^{i(n \tau-N) \sigma}\right)^{1 / \sigma}\left\|f \mid F_{p, q}^{s, \tau}\right\| \\
& \leq c\left|B_{J}\right|^{\tau}\left\|f \mid F_{p, q}^{s, \tau}\right\|,
\end{aligned}
$$

where the first inequality is obtained by Theorem 2.5 and the second inequality follows by taking $N>n \tau$. Taking $a \in(n / \min (p, q)$, s), then using again Lemma 3.6 to estimate (4.32) by

$$
c\left(\sum_{j \geq J^{+}} 2^{j s q}\left|\Delta_{j, J}^{*, a} f(x)\right|^{q}\right)^{1 / q} .
$$

This expression, by Theorem 2.5, in $L^{p}\left(B_{J}\right)$-norm is bounded by $c\left|B_{J}\right|^{\tau}\left\|f \mid F_{p, q}^{s, \tau}\right\|$. Hence we have for any $J \in \mathbb{Z}$ and any ball $B_{J}$ of $\mathbb{R}^{n}$ with radius $2^{-J}$

$$
\frac{1}{\left|B_{J}\right|^{\tau}}\left\|\left(H_{J}\right)^{1 / q}\left|L^{p}\left(B_{J}\right)\|\leq c\| f\right| F_{p, q}^{s, \tau}\right\|
$$

with some positive constant $c$ independent of $J$. From this it follows that

$$
\left\|f\left|F_{p, q}^{s, \tau}\left\|_{M} \leq c\right\| f\right| F_{p, q}^{s, \tau}\right\|
$$

for any $f \in F_{p, q}^{s, \tau}$.

Step 3. Let $\Psi$ be the function introduced in Definition 2.1 and in addition radial symmetric. We make use of an observation made by Nikol'skij [15] (see also [16]). We put

$$
\psi(x)=(-1)^{M+1} \sum_{i=0}^{M-1}(-1)^{i} C_{i}^{M} \Psi(x(M-i)) .
$$

The function $\psi$ satisfies $\psi(x)=1$ for $|x| \leq 1 / M$ and $\psi(x)=0$ for $|x| \geq 3 / 2$. Then, taking $\varphi_{0}(x)=\psi(x), \varphi_{1}(x)=\psi(x / 2)-\psi(x)$ and $\varphi_{j}(x)=\varphi_{1}\left(2^{-j+1} x\right)$ for $j=2,3, \ldots$, we obtain that $\left\{\varphi_{j}\right\}$ is a smooth dyadic resolution of unity. This yields that

$$
\sup _{B_{J}} \frac{1}{\left|B_{J}\right|^{\tau}}\left\|\left(\sum_{j \geq J^{+}} 2^{j s q}\left|\Delta_{j} f\right|^{q}\right)^{1 / q} \mid L^{p}\left(B_{J}\right)\right\|,
$$


Journal of Function Spaces and Applications

is a norm equivalent in $F_{p, q}^{s, \tau}$ (see Remark 2.3). Let us prove that

$$
\frac{1}{\left|B_{J}\right|^{\tau}}\left\|\left(\sum_{j \geq J^{+}} 2^{j s q}\left|\Delta_{j} f\right|^{q}\right)^{1 / q}\left|L^{p}\left(B_{J}\right)\|\leq c\| f\right| F_{p, q}^{s, \tau}\right\|_{M^{\prime}}
$$

for any ball $B_{J}$ of $\mathbb{R}^{n}$ with radius $2^{-J}$. First the left-hand side contains $\Delta_{0} f$ only when $J^{+}=0$. Then

$$
\left\|\Delta_{0} f\left|L^{p}\left(B_{J}\right)\left\|\leq \int_{\mathbb{R}^{n}}\right\| f\right| L^{p}\left(\bar{B}_{J}\right)\right\|\left|\mathcal{F}^{-1} \psi(y)\right| d y,
$$

where $\bar{B}_{J}$ is a ball centered at $x_{0}+y$ and of radius $2^{-J}$. Hence

$$
\begin{aligned}
\left\|\Delta_{0} f \mid L^{p}\left(B_{J}\right)\right\| & \leq c\left|B_{J}\right|^{\tau}\left\|f \mid L_{\tau}^{p}\right\| \\
& \leq c\left|B_{J}\right|^{\tau}\left\|f \mid F_{p, q}^{s, \tau}\right\|_{M^{\prime}}
\end{aligned}
$$

where we have used the fact that $\left|\bar{B}_{J}\right|=\left|B_{J}\right|$. Moreover, it holds for $x \in \mathbb{R}^{n}$ and $j=1,2, \ldots$

$$
\Delta_{j} f(x)=(-1)^{M+1} \int_{\mathbb{R}^{n}} \Delta_{2^{-j} v}^{M} f(x) \mathcal{F}^{-1} \tilde{\Psi}(v) d v,
$$

with $\tilde{\Psi}(\cdot)=\mathcal{F}^{-1} \Psi(\cdot)-2^{-n} \mathcal{F}^{-1} \Psi(\cdot / 2)$ (see [17, Theorem 3.1]). Now, for $j \in \mathbb{N}$ we write

$$
\begin{aligned}
& \int_{\mathbb{R}^{n}}\left|\Delta_{2^{-j} v}^{M} f(x)\right|\left|\mathcal{F}^{-1} \widetilde{\Psi}(v)\right| d v \\
& \quad=\int_{|v| \leq 1}\left|\Delta_{2^{-j} v}^{M} f(x)\right|\left|\mathcal{F}^{-1} \tilde{\Psi}(v)\right| d v+(-1)^{M+1} \int_{|v|>1}\left|\Delta_{2^{-j} v}^{M} f(x)\right|\left|\mathcal{F}^{-1} \widetilde{\Psi}(v)\right| d v .
\end{aligned}
$$

Then the estimate (4.42) is an obvious consequence of (4.44) and Lemma 3.4. Therefore,

$$
\left\|f\left|F_{p, q}^{s, \tau}\|\leq c\| f\right| F_{p, q}^{s, \tau}\right\|_{M^{\prime}}
$$

which completes the proof of Theorem 4.1.

Proof of Theorem 4.2. The first two steps closely follow the argument in [17, Theorem 3.1]. Step 1. Let $f \in B_{p, q}^{s, \tau}$. Since $s>0$, then we have

$$
\begin{aligned}
& \left\|f\left|L_{\tau}^{p}\left(\mathbb{R}^{n}\right)\left\|\leq \sum_{j \geq 0} 2^{-j s} 2^{j s}\right\| \Delta_{j} f\right| L_{\tau}^{p}\left(\mathbb{R}^{n}\right)\right\| \leq \underset{j \in \mathbb{N}_{0}}{ } 2^{j s}\left\|\Delta_{j} f \mid L_{\tau}^{p}\left(\mathbb{R}^{n}\right)\right\| \\
& \leq c\left\|f \mid B_{p, q}^{s, \tau}\right\| \text {. }
\end{aligned}
$$


Step 2. As in the proof of Theorem 4.1 we have

$$
\begin{aligned}
& \left(\int_{0}^{2^{-J^{+}+1}} t^{-s q} \sup _{|h| \leq t}\left\|\Delta_{h}^{M} f \mid L^{p}\left(B_{J}\right)\right\|^{q} \frac{d t}{t}\right)^{1 / q} \\
& \quad \leq c\left(\sum_{k \geq J^{+}} 2^{k s q} \sup _{|h| \leq 2^{-k+1}}\left\|\Delta_{h}^{M} f \mid L^{p}\left(B_{J}\right)\right\|^{q}\right)^{1 / q} .
\end{aligned}
$$

Let us estimate $\Delta_{h}^{M} \Delta_{j} f$. If $j \leq k$, then as in the proof Theorem 4.1, we have

$$
\left|\Delta_{h}^{M} \Delta_{j} f(x)\right| \leq c 2^{(j-k) M} \Delta_{j}^{*, a} f(x),
$$

for any $x \in B_{J},|h| \leq 2^{-k+1}$ and any $a>0$. If $j>k$ we have for $x \in \mathbb{R}^{n}$ and $|h| \leq 2^{-k+1}$

$$
\left|\Delta_{h}^{M} \Delta_{j} f(x)\right| \leq \sum_{m=0}^{M} C_{m}^{M}\left|\Delta_{j} f(x+(M-m) h)\right| .
$$

Hence we obtain for any $j>k$ and any $a>0$

$$
\begin{aligned}
\left\|\Delta_{h}^{M} \Delta_{j} f \mid L^{p}\left(B_{J}\right)\right\| & \leq \sum_{m=0}^{M} C_{m}^{M}\left\|\Delta_{j} f \mid L^{p}\left(\widetilde{B}_{J}\right)\right\| \\
& \leq \sum_{m=0}^{M} C_{m}^{M}\left\|\Delta_{j}^{*, a} f \mid L^{p}\left(\widetilde{B}_{J}\right)\right\|,
\end{aligned}
$$

where if $x_{0}$ the centre of $B_{J}$ then $x_{0}+(M-m) h$ is the centre of $\tilde{B}_{J}$. We remark also that by our assumption on $h$ and $k$ we have

$$
\left.\left|x-x_{0}\right| \leq \mid x-\left(x_{0}+(M-m) h\right)\right)|+(M-m)| h \mid<2^{-J}+M 2^{-k+1} \leq(2 M+1) 2^{-J},
$$

for any $x \in \widetilde{B}_{J}$. We denote $\widehat{B}_{J}$ the ball in $\mathbb{R}^{n}$ centred at $x_{0}$ and of radius $(2 M+1) 2^{-J}$. Since $L^{p}\left(\widehat{B}_{J}\right) \subset L^{p}\left(\widetilde{B}_{J}\right)$ and $L^{p}\left(\widehat{B}_{J}\right) \subset L^{p}\left(B_{J}\right)$, we get

$$
\begin{aligned}
\left\|\Delta_{h}^{M} f \mid L^{p}\left(B_{J}\right)\right\| \leq & c \sum_{j=0}^{J^{+}-1} 2^{(j-k) M}\left\|\Delta_{j}^{*, a} f\left|L^{p}\left(B_{J}\right)\left\|+c \sum_{j=J^{+}}^{k} 2^{(j-k) M}\right\| \Delta_{j}^{*, a} f\right| L^{p}\left(\widehat{B}_{J}\right)\right\| \\
& +c \sum_{j \geq k}\left\|\Delta_{j}^{*, a} f \mid L^{p}\left(\widehat{B}_{J}\right)\right\| .
\end{aligned}
$$

Here we put $\sum_{j=0}^{J^{+}-1} \cdots=0$ if $J^{+}=0$. Lemma 3.1 gives

$$
\begin{aligned}
\sum_{j=0}^{J^{+}-1} 2^{(j-k) M}\left\|\Delta_{j}^{*, a} f \mid L^{p}\left(B_{J}\right)\right\| & \leq c \sum_{j=0}^{J^{+}-1} 2^{(j-k) M+j(n / p-s-n \tau)-J n / p}\left\|f \mid B_{p, q}^{s, \tau}\right\| \\
& \leq c 2^{J(M-s-n \tau)-k M}\left\|f \mid B_{p, q}^{s, \tau}\right\| .
\end{aligned}
$$


The second inequality follows by our assumption on $s$ and $\tau$. Since $0<s<M$, then we can apply Lemma 3.6 to estimate (4.49) by

$$
\begin{aligned}
& c\left|B_{J}\right|^{\tau}\left\|f\left|B_{p, q}^{s, \tau}\|\|\left\{2^{\left(k-J^{+}\right)(s-M)}\right\}_{k \geq J^{+}}\right| \ell_{q, J^{+}}^{0}\right\|+c\left(\sum_{j \geq J^{+}} 2^{j s q}\left\|\Delta_{j}^{*, a} f \mid L^{p}\left(\widehat{B}_{J}\right)\right\|^{q}\right)^{1 / q} \\
& \leq c\left|B_{J}\right|^{\tau}\left\|f \mid B_{p, q}^{s, \tau}\right\|,
\end{aligned}
$$

where we have used Theorem 2.4, combined with Remark 2.6, and the equation $\left|\widehat{B}_{J}\right|=(2 M+$ $1)^{n}\left|B_{J}\right|$.

Step 3. First this step in [17, Theorem 3.1] contains a gap, but using the same arguments given in Step 3 in the proof of Theorem 4.1 (with Lemma 3.5 in place of Lemma 3.4), we can prove that

$$
\left\|f\left|B_{p, q}^{s, \tau}\|\leq c\| f\right| B_{p, q}^{s, \tau}\right\|_{M}
$$

This ends the proof of Theorem 4.2.

Finally we study, in addition, the case $\tau \in[1 / p, \infty)$. Under this condition we can restrict $\sup _{B_{J}}$ in the definition of $B_{p, q}^{s, \tau}$ and $F_{p, q}^{s, \tau}$ to a supremum taken with respect to balls $B_{J}$ of $\mathbb{R}^{n}$ with radius $2^{-J}$ and $J \in \mathbb{N}_{0}$.

Lemma 4.4. Let $s \in \mathbb{R}, \tau \in[1 / p, \infty)$ and $0<q \leq \infty$.

Let $0<p \leq \infty$. A tempered distribution $f$ belongs to $B_{p, q}^{s, \tau}$ if and only if

$$
\left\|f \mid B_{p, q}^{s, \tau}\right\|^{\#}=\sup _{B_{J}} \frac{1}{\left|B_{J}\right|^{\tau}}\left(\sum_{j \geq J} 2^{j s q}\left\|\Delta_{j} f \mid L^{p}\left(B_{J}\right)\right\|^{q}\right)^{1 / q}<\infty,
$$

where the supremum is taken over all $J \in \mathbb{N}_{0}$ and all balls $B_{J}$ of $\mathbb{R}^{n}$ with radius $2^{-J}$. Furthermore, the quasinorms $\left\|f \mid B_{p, q}^{s, \tau}\right\|^{\#}$ and $\left\|f \mid B_{p, q}^{s, \tau}\right\|$ are equivalent.

Let $0<p<\infty$. A tempered distribution $f$ belongs to $F_{p, q}^{s, \tau}$ if and only if

$$
\left\|f\left|F_{p, q}^{s, \tau}\left\|^{\#}=\sup _{B_{J}} \frac{1}{\left|B_{J}\right|^{\tau}}\right\|\left(\sum_{j \geq J} 2^{j s q}\left|\Delta_{j} f\right|^{q}\right)^{1 / q}\right| L^{p}\left(B_{J}\right)\right\|<\infty,
$$

where the supremum is taken over all $J \in \mathbb{N}_{0}$ and all balls $B_{J}$ of $\mathbb{R}^{n}$ with radius $2^{-J}$. Furthermore, the quasinorms $\left\|f \mid F_{p, q}^{s, \tau}\right\|^{\#}$ and $\left\|f \mid F_{p, q}^{s, \tau}\right\|$ are equivalent.

Proof. For each $J \in \mathbb{Z}$ and $m=\left(m_{1}, \ldots, m_{n}\right) \in \mathbb{Z}^{n}$, set

$$
Q_{J, m}=\left\{x=\left(x_{1}, \ldots, x_{n}\right) \in \mathbb{R}^{n}: m_{i} \leq 2^{J} x_{i}<\left(m_{i}+1\right), i=1,2, \ldots, n\right\} .
$$

This lemma for $Q_{J, m}$ in place of $B_{J}$ is given in [9, Lemma 2.2]. By the properties of the dyadic cubes, there exists $v, k \in \mathbb{N}$ not depending on $J$ such that

$$
Q_{J, m} \subset \cup_{l=1}^{v} B_{J}^{l}, \quad B_{J} \subset \cup_{l=1}^{k} Q_{J}^{l} .
$$


Here $B_{J}^{l}$ is a ball of center $2^{-J}$ and $Q_{J}^{l}$ is a dyadic cube of side length $2^{-J}$. The proof of this result is an obvious consequence of the previous embeddings, Lemma 2.2 of [9] and Remark 2.3.

Defining for $1 \leq p \leq \infty$

$$
M_{p}(f)=\sup _{B_{0}}\left(\int_{B_{0}}|f(x)|^{p} d x\right)^{1 / p}
$$

where the supremum is taken over all balls $B_{0}$ of $\mathbb{R}^{n}$ with radius 1 .

Theorem 4.5. Let $1 \leq p<\infty, 0<q \leq \infty, \tau \in[1 / p, \infty), M \in \mathbb{N}$ and $n / \min (p, q)<s<$ $M-n \tau+n / p$. Then

$$
\left\|f\left|F_{p, q}^{s, \tau}\left\|_{M}^{\#}=M_{p}(f)+\sup _{B_{J}} \frac{1}{\left|B_{J}\right|^{\tau}}\right\|\left(\int_{0}^{2^{-J+1}} t^{-s q} \sup _{|h| \leq t}\left|\Delta_{h}^{M} f(\cdot)\right|^{q} \frac{d t}{t}\right)^{1 / q}\right| L^{p}\left(B_{J}\right)\right\|
$$

is an equivalent quasinorm in $F_{p, q}^{s, \tau}$. Here the supremum is taken over all $J \in \mathbb{N}_{0}$ and all balls $B_{J}$ of $\mathbb{R}^{n}$ with radius $2^{-J}$.

Theorem 4.6. Let $1 \leq p \leq \infty, 0<q \leq \infty, \tau \in[1 / p, \infty), M \in \mathbb{N}$ and $0<s<M-n \tau+n / p$. Then

$$
\left\|f \mid B_{p, q}^{s, \tau}\right\|_{M}^{\#}=M_{p}(f)+\sup _{B_{J}} \frac{1}{\left|B_{J}\right|^{\tau}}\left(\int_{0}^{2^{-J+1}} t^{-s q} \sup _{|h| \leq t}\left\|\Delta_{h}^{M} f \mid L^{p}\left(B_{J}\right)\right\|^{q} \frac{d t}{t}\right)^{1 / q}
$$

is an equivalent quasinorm in $B_{p, q}^{s, \tau}$. Here the supremum is taken over all $J \in \mathbb{N}_{0}$ and all balls $B_{J}$ of $\mathbb{R}^{n}$ with radius $2^{-J}$.

Proof. We will prove only Theorem 4.5 . The proof of Theorem 4.6 is similar. We employ the same notations given in the proof of Theorem 4.1.

Step 1. Let $f \in F_{p, q}^{s, \tau}$. Since $s>0$, then we have

$$
\begin{aligned}
M_{p}(f) & \leq \sum_{j \geq 0} 2^{-j s} 2^{j s} M_{p}\left(\Delta_{j} f\right) \leq c \sup _{B_{0}, j \in \mathbb{N}_{0}} 2^{j s}\left\|\Delta_{j} f \mid L^{p}\left(B_{0}\right)\right\| \\
& \leq c\left\|f \mid F_{p, q}^{s, \tau}\right\|^{\#} .
\end{aligned}
$$

Step 2. As in the proof of Theorem 4.1, there is a constant $c>0$ independent of $J$ such that

$$
\begin{aligned}
\frac{1}{\left|B_{J}\right|^{\tau}}\left\|\left(H_{J}\right)^{1 / q} \mid L^{p}\left(B_{J}\right)\right\| & \leq c\left\|f \mid F_{p, q}^{s, \tau}\right\| \\
& \leq c\left\|f \mid F_{p, q}^{s, \tau}\right\|^{\#},
\end{aligned}
$$

by Lemma 4.4 . 
Step 3. The left-hand side in (4.42) (with $J^{+}=J$ ) contains $\Delta_{0} f$ only when $J=0$. Then

$$
\begin{aligned}
\left\|\Delta_{0} f \mid L^{p}\left(B_{0}\right)\right\| & \leq \int_{\mathbb{R}^{n}}\left\|f\left|L^{p}\left(\bar{B}_{0}\right) \|\right| \mathcal{F}^{-1} \psi(y) \mid d y\right. \\
& \leq c M_{p}(f) .
\end{aligned}
$$

We recall that for $x \in \mathbb{R}^{n}$ and $j=1,2, \ldots$

$$
\begin{aligned}
\Delta_{j} f(x) & =(-1)^{M+1} \int_{\mathbb{R}^{n}} \Delta_{2^{-j} v}^{M} f(x) \mathcal{F}^{-1} \tilde{\Psi}(v) d v \\
& =(-1)^{M+1} \int_{|v| \leq 1} \Delta_{2^{-j} v}^{M} f(x) \mathcal{F}^{-1} \tilde{\Psi}(v) d v+(-1)^{M+1} \int_{|v|>1} \Delta_{2^{-j} v}^{M} f(x) \mathcal{F}^{-1} \tilde{\Psi}(v) d v
\end{aligned}
$$

As in the proof of Lemma 3.4, we can prove that

$$
\begin{gathered}
\left\|\left(\sum_{j \geq J} 2^{j s q}\left(\int_{|v| \leq 1}\left|\Delta_{2^{-j} v}^{M} f(\cdot)\right| d v\right)^{q}\right)^{1 / q}\left|L^{p}\left(B_{J}\right)\left\|\leq c\left|B_{J}\right|^{\tau}\right\| f\right| F_{p, q}^{s, \tau}\right\|_{M^{\prime}}^{\#} \\
\left\|\left(\sum_{j \geq J} 2^{j s q}\left(\int_{|v|>1}\left|\Delta_{2^{-j} v}^{M} f(\cdot) \omega(v)\right| d v\right)^{q}\right)^{1 / q}\left|L^{p}\left(B_{J}\right)\left\|\leq c\left|B_{J}\right|^{\tau}\right\| f\right| F_{p, q}^{s, \tau}\right\|_{M^{\prime}}^{\#}
\end{gathered}
$$

for any $J \in \mathbb{N}$ any ball $B_{J}$ of $\mathbb{R}^{n}$ with radius $2^{-J}$ and any $\omega \in \mathcal{S}\left(\mathbb{R}^{n}\right)$. The proof is completed.

Remark 4.7. Recently, Yang and Yuan [18, Theorem 2] proved that

$$
\begin{array}{ll}
F_{p, q}^{s, \tau}=F_{\infty, \infty}^{s+n(\tau-1 / p)}, & 0<p<\infty, s \in \mathbb{R}, \\
B_{p, q}^{s, \tau}=B_{\infty, \infty}^{s+n(\tau-1 / p)}, & 0<p \leq \infty, s \in \mathbb{R},
\end{array}
$$

if $\tau>1 / p, 0<q \leq \infty$ or if $\tau=1 / p$ and $q=\infty$. Under these conditions the study of the Triebel-Lizorkin-type space $F_{p, q}^{s, \tau}$ and the Besov-type space $B_{p, q}^{s, \tau}$ is not interest.

\section{Acknowledgments}

The author would like to thank the referee for his very carefully reading and also his many careful and valuable remarks, which improve, the results and the presentation of this paper.

\section{References}

[1] H. Triebel, Theory of Function Spaces, Birkhäuser, Basel, Switzerland, 1983.

[2] H. Triebel, Theory of Function Spaces. II, Birkhäuser, Basel, Switzerland, 1992. 
[3] H. Triebel, Theory of Function Spaces. III, Birkhäuser, Basel, Switzerland, 2006.

[4] A. El Baraka, "An embedding theorem for Campanato spaces," Electronic Journal of Differential Equations, vol. 66, pp. 1-17, 2002.

[5] A. El Baraka, "Littlewood-Paley characterization for Campanato spaces," Journal of Function Spaces and Applications, vol. 4, no. 2, pp. 193-220, 2006.

[6] S. Campanato, "Proprietà di hölderianità di alcune classi di funzioni," vol. 17, pp. 175-188, 1963.

[7] S. Campanato, "Proprietà di una famiglia di spazi funzionali," Annali della Scuola Normale Superiore di Pisa, vol. 18, pp. 137-160, 1964.

[8] D. Drihem, "Some embeddings and equivalent norms of the $\mathcal{L}_{p, q}^{\lambda, s}$ spaces," Functiones et Approximatio Commentarii Mathematici, vol. 41, no. 1, pp. 15-40, 2009.

[9] W. Yuan, W. Sickel, and D. Yang, Morrey and Campanato Meet Besov, Lizorkin and Triebel, vol. 2005 of Lecture Notes in Mathematics, Springer, Berlin, Germany, 2010.

[10] D. Yang and W. Yuan, "A new class of function spaces connecting Triebel-Lizorkin spaces and $Q$ spaces," Journal of Functional Analysis, vol. 255, no. 10, pp. 2760-2809, 2008.

[11] D. Yang and W. Yuan, "New Besov-type spaces and Triebel-Lizorkin-type spaces including $Q$ spaces," Mathematische Zeitschrift, vol. 265, no. 2, pp. 451-480, 2010.

[12] D. Yang and W. Yuan, "Characterizations of Besov-type and Triebel-Lizorkin-type spaces via maximal functions and local means," Nonlinear Analysis, vol. 73, no. 12, pp. 3805-3820, 2010.

[13] Y. Sawano, D. Yang, and W. Yuan, "New applications of Besov-type and Triebel-Lizorkin-type spaces," Journal of Mathematical Analysis and Applications, vol. 363, no. 1, pp. 73-85, 2010.

[14] D. Drihem, La multiplication ponctuelle dans les espaces de Besov et Triebel-Lizorkin, Ph.D. thesis, Batna University, 2007.

[15] S. M. Nikol'skij, Approximation of Function of Several Variables and Imbedding Theorem, Springer, Berlin, Germany, 1975.

[16] W. Sickel, "On pointwise multipliers for $F_{p, q}^{s}\left(\mathbb{R}^{n}\right)$ in case $\sigma_{p, q}<s<n / p$," Annali di Matematica Pura ed Applicata, vol. 176, pp. 209-250, 1999.

[17] D. Drihem, "Some characterizations of function spaces connecting $\mathcal{L}^{2, \alpha}$ spaces," Revista Matemática Complutense, vol. 24, no. 2, pp. 323-333, 2011.

[18] D. Yang and W. Yuan, "Relations among besov-type spaces, triebel-lizorkin-type spaces and generalized carleson measure spaces," Applicable Analysis, vol. 2011, 13 pages, 2011. 


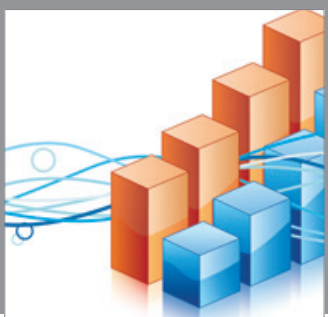

Advances in

Operations Research

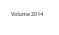

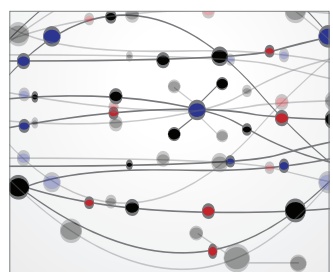

\section{The Scientific} World Journal
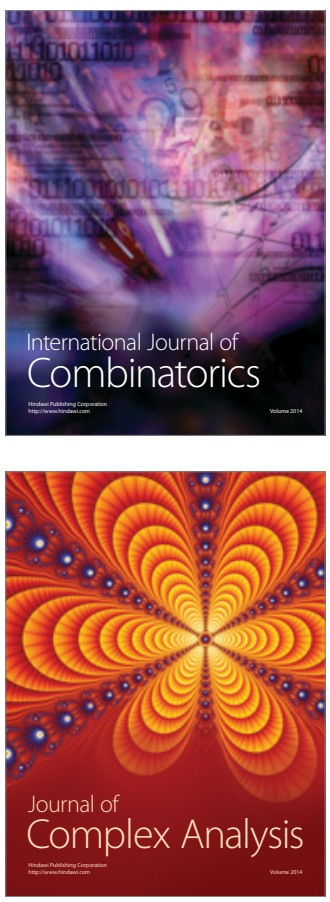

International Journal of

Mathematics and

Mathematical

Sciences
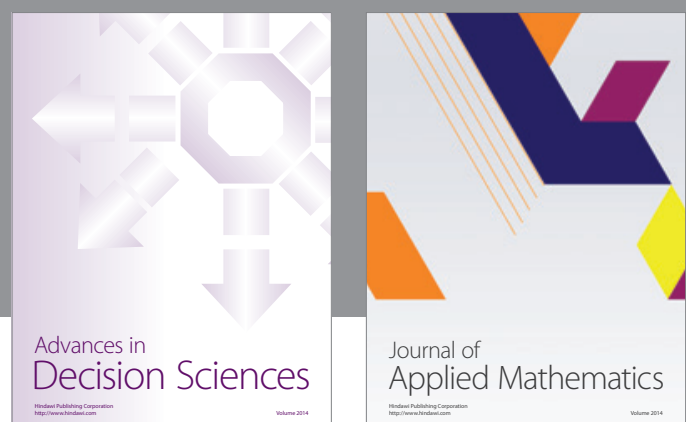

Journal of

Applied Mathematics
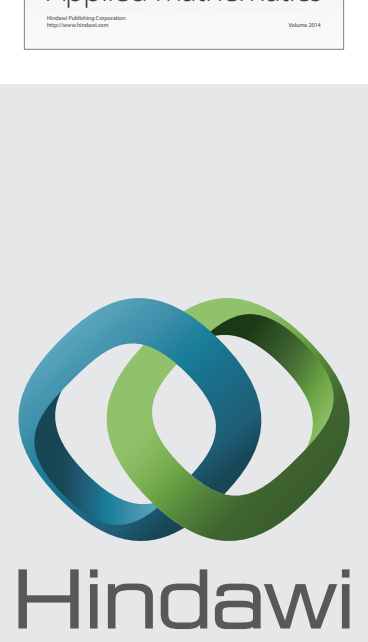

Submit your manuscripts at http://www.hindawi.com
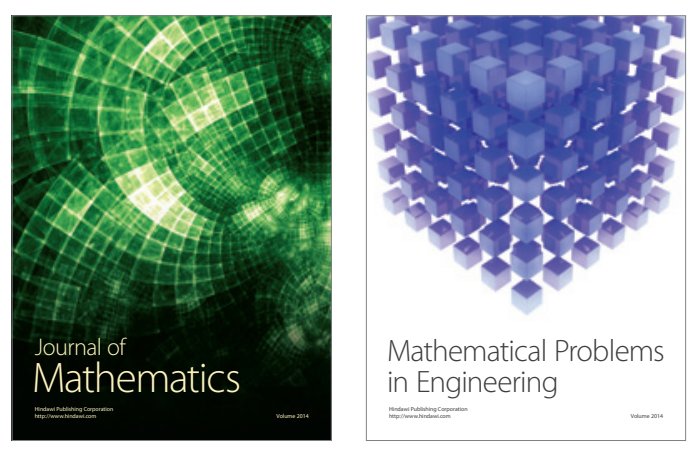

Mathematical Problems in Engineering
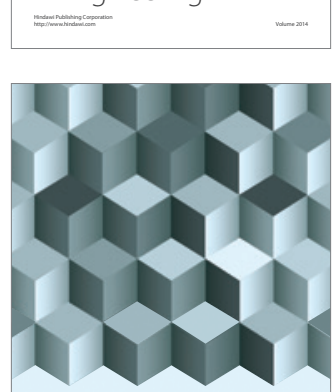

Journal of

Function Spaces
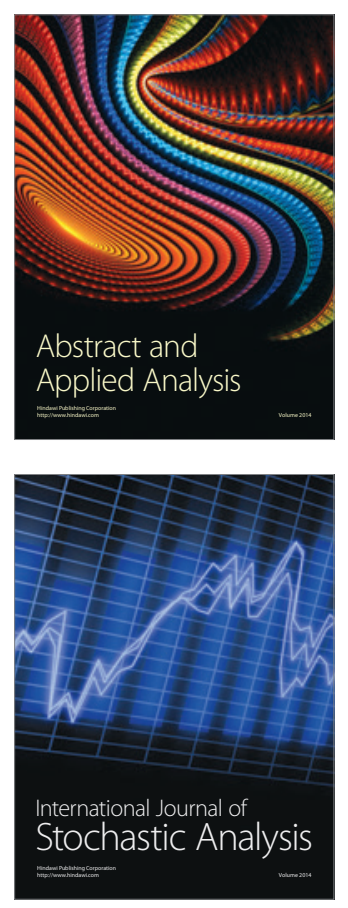

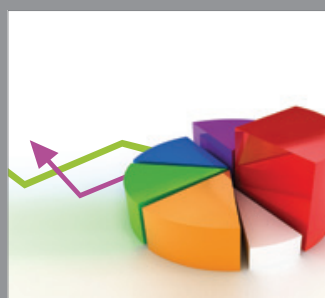

ournal of

Probability and Statistics

Promensencen
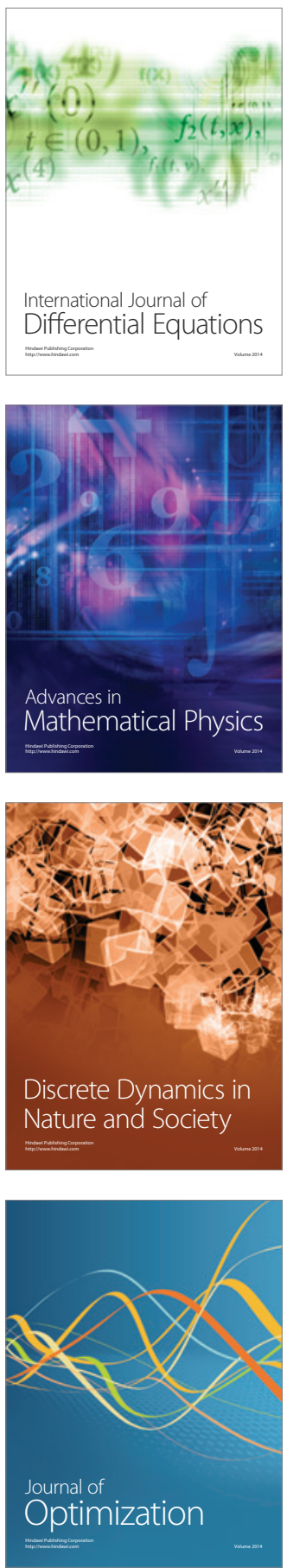